\title{
Besonderheiten des schnellenden Fingers im Kindesalter
}

\section{Specific Characteristics of Trigger Finger in Children}

\author{
Autoren \\ K. Kalb , D. Möllmeier², W. Hülsemann \\ Institute \\ ${ }^{1}$ Rhön-Klinikum AG, Klinik für Handchirurgie, Bad Neustadt/Saale \\ ${ }^{2}$ Berufsgenossenschaftliches Unfallkrankenhaus, Handchirurgie, Plastische und Mikrochirurgie, Hamburg \\ ${ }^{3}$ Katholisches Kinderkrankenhaus Wilhelmstift, Handchirurgie, Hamburg
}

\section{Schlüsselwörter \\ - Tendovaginosis stenosans im Kindesalter \\ - Resektion FDS-Zügel \\ - Schnellender Finger}

Key words

- trigger finger in children

- partial FDS resection

trigger finger eingereicht 26.09.2015

akzeptiert $\quad 31.12 .2015$

Bibliografie

Dol http://dx.doi.org/ 10.1055/s-0042-100458 Handchir Mikrochir Plast Chir 2016; 48: 33-40 (c) Georg Thieme Verlag KG Stuttgart · New York ISSN 0722-1819

Correspondence Dr. Karlheinz Kalb Rhön-Klinikum AG Klinik für Handchirurgie 97615 Bad Neustadt Bad Neustadt/Saale 97616 DrKalb@web.de

\section{Zusammenfassung}

\section{$\nabla$}

Hintergrund: Die Tendovaginosis stenosans der Finger ist bei Kindern selten und weist relevante Besonderheiten im Vergleich zum häufigeren Befall des Daumens und zu der sehr häufigen Erkrankung im Erwachsenenalter auf.

Patienten und Methoden: Bei der Analyse von Kindern mit Operation einer Tendovaginosis stenosans der Finger in 2 spezialisierten handchirurgischen Einrichtungen in einem Zeitraum von 12 Jahren fanden wir insgesamt 42 Patienten mit 63 erkrankten Fingern. Bei 12 dieser Kinder mit insgesamt 17 betroffenen Fingern im Alter von durchschnittlich 3,7 (0,6-10,2) Jahren waren aufgrund des intraoperativen Befundes bei fortbestehendem Schnappen nach Spaltung des Ringbandes A1 zusätzliche Maßnahmen, in erster Linie die Resektion eines Zügels der oberflächlichen Beugesehne erforderlich, wobei bei einem Kind bei einem weiteren gleichzeitig operierten Finger die A1-Ringbandspaltung genügte. Neben einer Auswertung der Krankenunterlagen wurden die klinischen Ergebnisse in dieser Untergruppe telefonisch durchschnittlich 4,8 (1,2$11,8)$ Jahre postoperativ erfragt. Es wurden verbliebene Funktionseinschränkungen, Schmerzen, Zufriedenheit und Komplikationen erfasst.

Ergebnisse: Alle Patienten, die mit einer Spaltung des Ringbandes A1 und einer isolierten Resektion eines FDS-Zügels behandelt wurden, hatten ein ausgezeichnetes Ergebnis. Bei einer Patientin kam es zu einem Rezidiv und eine weitere Patientin entwickelte eine Beugekontraktur des Mittelgelenkes. In diesen Fällen war die Weitung der Sehnenscheide neben der A1-Ringbandspaltung durch eine Erweiterungsplastik des Ringbandes A2 erfolgt.

Schlussfolgerung: Führt die Spaltung des Ringbandes A1 bei der Tendovaginosis stenosans eines Fingers beim Kind nicht zu einem freien Sehnengleiten, so lässt sich dies durch zusätzliche Resektion eines FDS-Zügels dauerhaft erfolgreich erzielen.

\section{Abstract \\ $\nabla$}

Background: Trigger finger in children is a rare condition with relevant differences to the more frequent trigger thumb in children and the very frequent trigger finger in adults.

Patients and methods: In a retrospective analysis of children who underwent surgery for trigger fingers in 2 specialised centres, we evaluated 42 children with a total of 63 trigger fingers. Due to persistent triggering after release of the A1 pulley based on intraoperative findings in 12 children with a mean age of 3.7 years ranging from 0.6 to 10.2 years and a total of 17 trigger fingers, additional measures after the release of the A1 pulley were required, mainly the resection of one limb of the sublimis tendon. In one of these children another simultaneously operated finger required the release of the A1 pulley alone. We performed a retrospective chart review of these patients and evaluated the results after a mean follow-up time of 4.8 years ranging from 1.2 to 11.8 years by phone call. Functional impairments, persistent pain, satisfaction and complications were monitored.

Results: All patients treated with A1 pulley release and resection of one limb of the sublimis tendon had excellent results. One patient had a recurrence and another patient developed a contracture of the PIP joint. In both patients, the widening of the tendon sheath had been done by $\mathrm{A} 2$ pulley enlargement in addition to the $\mathrm{A} 1$ pulley release.

Conclusions: Persistent triggering of a finger following the release of the A1 pulley in a child can be successfully treated by resection of one limb of the sublimis tendon. 


\section{Einleitung \\ $\nabla$}

Die operative Behandlung eines schnellenden Fingers gilt als chirurgischer Bagatelleingriff. Sie zählt zu den häufigsten Operationen an der Hand und eignet sich gut als Anfängeroperation, wenn sie unter sachgerechter Anleitung erfolgt. Die Spaltung des Ringbandes A1 ist beim Erwachsenen die Therapie der Wahl und beseitigt die klinische Symptomatik fast immer. Dass im Kindesalter die Erkrankung hauptsächlich am Daumen auftritt, wo sie typischerweise nicht mit einem Schnappphänomen, sondern oft mit einer fixierten Beugestellung im Endgelenk einhergeht, ist Allgemeingut. Nicht geläufig ist dagegen, dass die Erkrankung im Kindesalter nicht nur am Daumen sondern auch an den Fingern auftreten kann. Sie ist hier allerdings deutlich seltener. Die Literaturangaben schwanken zwischen 7 und 14\% [1] im Verhältnis zum Pollex rigidus. Dieses relativ seltene Krankheitsbild weist Besonderheiten im Vergleich zur Tendovaginosis stenosans im Erwachsenenalter auf. Seit den Publikationen von Cardon et al. [1] sowie Tordai et al. [2] wissen wir, dass die alleinige Spaltung des Ringbandes A1 an den Fingern II bis V im Kindesalter häufig unzureichend ist. Cardon et al. [1] geben an, dass in ihrem Krankengut bei $44 \%$ der operierten Kinder die Spaltung des Ringbandes A1 das Schnappphänomen nicht beseitigen konnte. Sie empfahlen neben der Spaltung des Ringbandes A1 die zusätzliche Resektion eines FDS-Zügels als definitive Therapie mit hoher Erfolgsrate. Vor diesem Hintergrund wollten wir unser Patientengut retrospektiv analysieren und speziell die Patienten, bei denen neben der obligaten Spaltung des Ringbandes A1 zusätzlich eine Resektion eines FDS-Zügels oder weitere Maßnahmen erfolgten, evaluieren. Ziel der Arbeit ist die Entwicklung eines standardisierten Vorgehens im eigenen Bereich.

\section{Patienten und Methoden \\ $\nabla$}

Im Zeitraum von Januar 2003 bis Dezember 2014 wurden in 2 spezialisierten handchirurgischen Zentren insgesamt 42 Kinder an 63 Fingern (davon 8 Kinder mit 11 Fingern in der Klinik des Erstautors und 34 Kinder mit 52 Fingern in der Klinik der Co-Autoren) mit einem Alter von maximal 10 Jahren wegen einer Tendovaginosis stenosans der Finger operiert. Erkrankungen des Daumens wurden ausgeschlossen.

Bei insgesamt 12 dieser Kinder erfolgten aufgrund eines fortbestehenden Schnappens nach der einfachen Spaltung des Ringbandes A1 17-mal weitere operative Maßnahmen, wobei bei einer dieser Patientinnen, bei der 3 Finger operiert wurden, ein Finger durch eine alleinige Spaltung des Ringbandes A1 behandelt werden konnte, obwohl auch hier eine Verdickung der FDS-Sehne beobachtet wurde. Diese 12 Kinder bilden die Grundlage unserer retrospektiven Nachuntersuchung $\bullet$ Tab. 1. Bei einem dieser Patienten handelte es sich bereits um einen Rezidiveingriff an 2 Fingern $\bullet$ Tab. 1

Das Alter dieser 12 Patienten (3 aus der Klinik des Erstautors und 9 aus der Klinik der Co-Autoren) lag zwischen 0,6 und 10,2 Jahren (Mittelwert: 3,7 Jahre). Zusätzliche Maßnahmen waren erforderlich: 3-mal am Zeigefinger, 7-mal am Mittelfinger, 6-mal am Ringfinger und 1-mal am Kleinfinger.

Die Analyse der Ergebnisse dieser Patientengruppe erfolgte im Rahmen einer telefonischen Befragung unter Einbeziehung der Eltern über verbliebene oder postoperativ wieder aufgetretene Funktionsstörungen wie Schnappphänomene, Kontrakturen oder sonstige Probleme der operierten Finger. Bei der telefoni- schen Befragung waren die Patienten zwischen 3,5 und 15,1 (Mittel 8,2) Jahre alt. Seit der Operation waren im Mittel 4,8 $(1,2-11,8)$ Jahre vergangen. Eine Patientin konnte nicht erreicht werden.

\section{Ergebnisse}

$\nabla$

An beiden Kliniken zusammen wurde an 10 Fingern bei $6 \mathrm{~Pa}-$ tienten eine A1-Ringbandspaltung und die Resektion eines Schenkels der Sehne des Flexor digitorum superficialis durchgeführt. Neunmal wurde der ulnare und 1-mal der radiale Zügel reseziert. Bei einem Kind erfolgte an 2 Fingern eine A1-Ringbandspaltung mit zusätzlicher Synovialektomie. An 4 Fingern bei 4 Patienten erfolgte neben der A1- Ringbandspaltung einmal eine partielle proximale Einkerbung des Ringbandes A2 unter Erhalt der Stabilität sowie 3-mal eine Erweiterungsplastik des Ringbandes A2, 1-mal in Kombination mit einer Resektion eines radialen FDS-Zügels. Ein Patient erhielt neben der A1-Ringbandspaltung nur die Resektion eines kolbenartig aufgetrieben Knotens der Sehne des Flexor digitorum superficalis.

Wie in Tabelle1 dargestellt zeigten sich neben der Tendovaginosis stenosans intraoperativ an allen 18 operierten Fingern weitere pathologische Befunde, allerdings war 1-mal die alleinige A1-Ringbandspaltung zur Beseitigung des Schnappphänomens ausreichend (linker Mittelfinger bei Patient 4). 3 Finger hatten eine spindelförmige Verdickung der Sehne des Flexor digitorum superficialis. Bei 3 Fingern fand sich nur eine spindelförmige Verdickung des ulnaren Schenkels, bei 2 Fingern eine des radialen Schenkels der Sehne des Flexor digitorum superficialis (FDS). Ein ulnarer Schenkel der FDS war bei dem einen Patienten mit Rezidiveingriff fibrotisch verändert und hierdurch verdickt. An 7 Fingern zeigte sich intraoperativ eine fusiforme Verdickung der Sehne des Flexor digitorum profundus (FDP). Die kolbenartige Auftreibung eines FDS-Zügels eines Fingers erwies sich histopathologisch als narbenartige Metaplasie traumatischer Genese. Eine fixierte Blockierung fanden wir bei einem Patienten.

11 der 12 Patienten bzw. deren Eltern konnten telefonisch erreicht werden und standen zu einer Befragung zur Verfügung. Hierbei wurde bei 14 von 17 Fingern (82,4\%) ein komplikationsloser Heilungsverlauf berichtet. Die Funktion der Finger war vollständig wieder hergestellt In der aktuellen Befragung beschrieb 1 Patient ein erneutes Schnappen ohne Bewegungslimitierung oder Schmerzen der Finger. Eine weitere Patientin stellte sich nach der telefonischen Befragung in der Sprechstunde vor. Leider mussten wir bei ihr eine Beugekontraktur von $90^{\circ}($ !) im Mittelgelenk des linken Ringfingers feststellen ( $\bullet$ Abb. 1). Ursächlich imponierte ein Bogensehnenphänomen nach A2-Ringbanderweiterungsplastik und FDS-Zügelresektion. Alle Kinder verneinten aktuell Schmerzen in Ruhe sowie unter Belastung der operativ versorgten Finger.

\section{Diskussion \\ $\nabla$}

Das Hauptanliegen der vorliegenden Arbeit ist es, den Leser für die Besonderheiten der Tendovaginosis stenosans der Finger im Kindesalter zu sensibilisieren. Dabei kann an den Fingern sowohl ein Schnappphänomen als auch eine fixierte Fehlstellung vorliegen. Auch mehrere Finger können ebenso betroffen sein wie beide Hände [3], wobei die Symptomatik nicht unmittelbar nach der Geburt auftritt. Der schnellende Finger im Erwachse- 


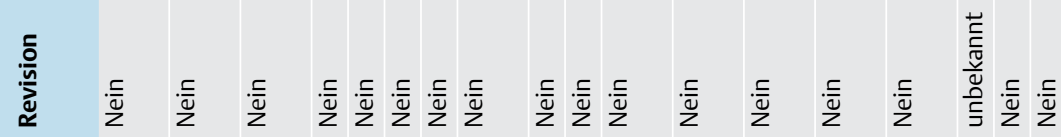

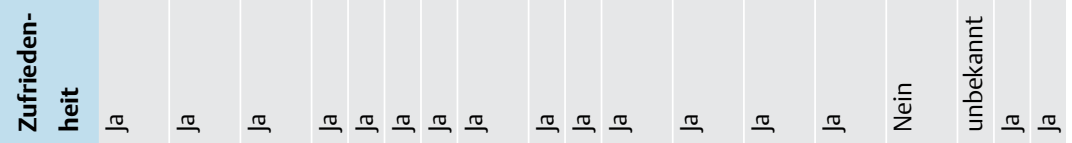

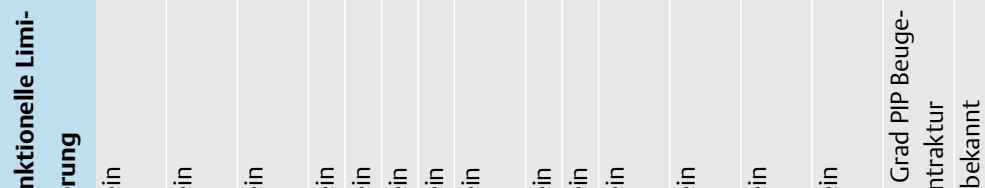

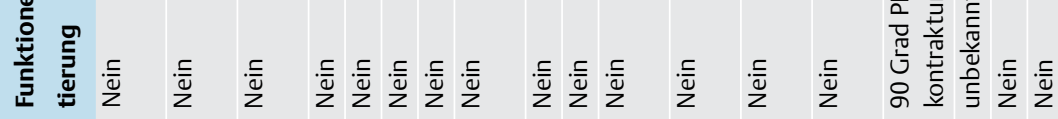

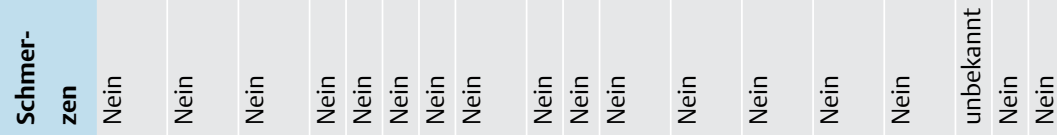

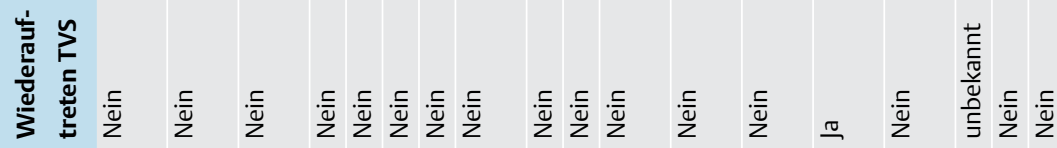
咅离㐫

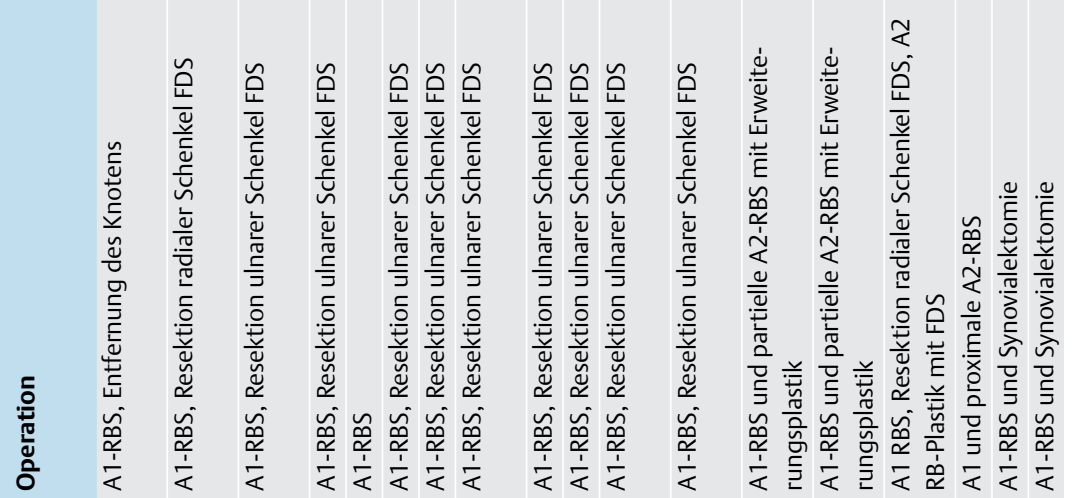

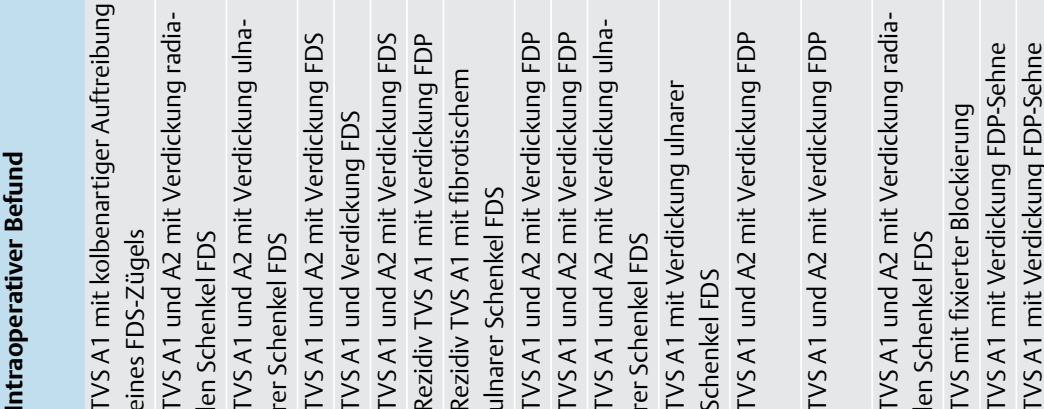

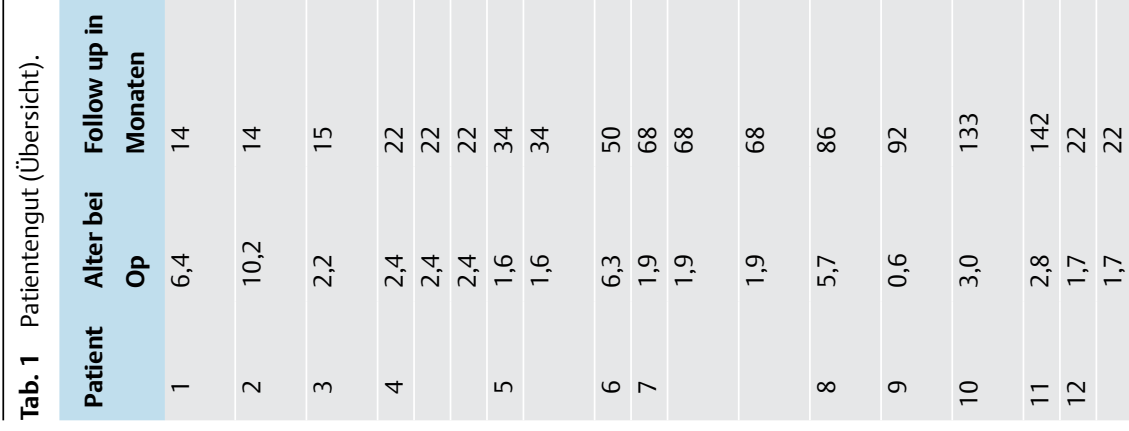




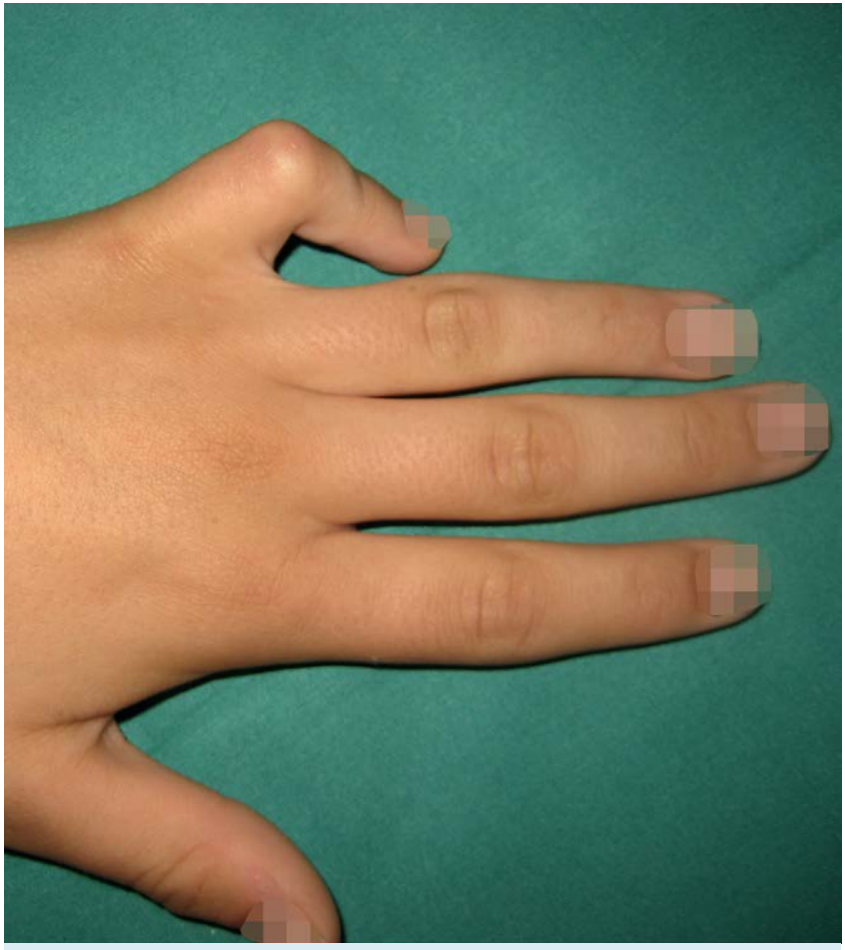

Abb. 1 Mittelgelenksbeugekontraktur nach TVS am Kleinfinger.

nenalter ist extrem häufig und kann - von seltenen Ausnahmefällen abgesehen - zuverlässig mit einer Spaltung des Ringbandes A1 beseitigt werden. Der naheliegende Analogieschluss auf das sehr seltene Krankheitsbild der Tendovaginose der Finger im Kindesalter ist unzulässig und gefährlich, da hier eine komplexere Ätiologie und Pathophysiologie zugrunde liegen. Dies schlägt sich in einer erheblichen Rezidiv- bzw. Persistenzrate der Tendovaginose bei einfacher A1-Ringbandspaltung nieder. Wird der Handchirurg angesichts der Seltenheit des Krankheitsbildes damit konfrontiert, besteht die Gefahr der Bagatellisierung und Fehleinschätzung. Wird die Symptomatik durch einen operativen Eingriff nicht beseitigt, gerät der Behandler in erhebliche Erklärungsnot.

In unserer Untersuchung fanden wir bei einem erheblichen Anteil unserer Patienten intraoperativ eine komplexe Pathologie, die eine einfache A1-Ringbandspaltung definitiv unzureichend erscheinen ließ. Beobachtet wurden bspw. lokale Synovialitiden ( $\odot$ Abb. 2) und knotige Verdickungen in der oberflächlichen ( $\bullet$ Abb. 3) und/oder tiefen Beugesehne ( $\bullet$ Abb. 4), wobei auch nach Spaltung des Ringbandes A1 ein Schnappen verblieben war. Alle Patienten, bei denen neben einer Spaltung des Ringbandes A1 eine isolierte Resektion eines FDS-Zügels erfolgte, hatten später keine weiteren Probleme mehr. Analysiert man die 2 Patienten mit persistierender Symptomatik, so lässt sich in beiden Fällen eine konkrete Ursache erkennen. Im einen Fall war das Missverhältnis zwischen Weite des osteofibrösen Gleitkanals und seinem Inhalt nicht durch eine Resektion eines FDS-Zügels, sondern durch eine Erweiterungsplastik des Ringbandes A2 therapiert worden. Bei der anderen Patientin, die die gravierende Beugekontraktur im Mittelgelenk entwickelte ( $\bullet$ Abb. 1), war neben der Spaltung des Ringbandes A1 und der Resektion eines FDS-Zügels zusätzlich eine Erweiterungsplastik des Ringbandes A2 erfolgt. Letztlich führte also die nur ausnahmsweise vorgenommene A2-Ringbanderweiterungsplastik in unserem Krankengut bei 2 von 3 Patienten zu Problemen. Prinzipiell kann das

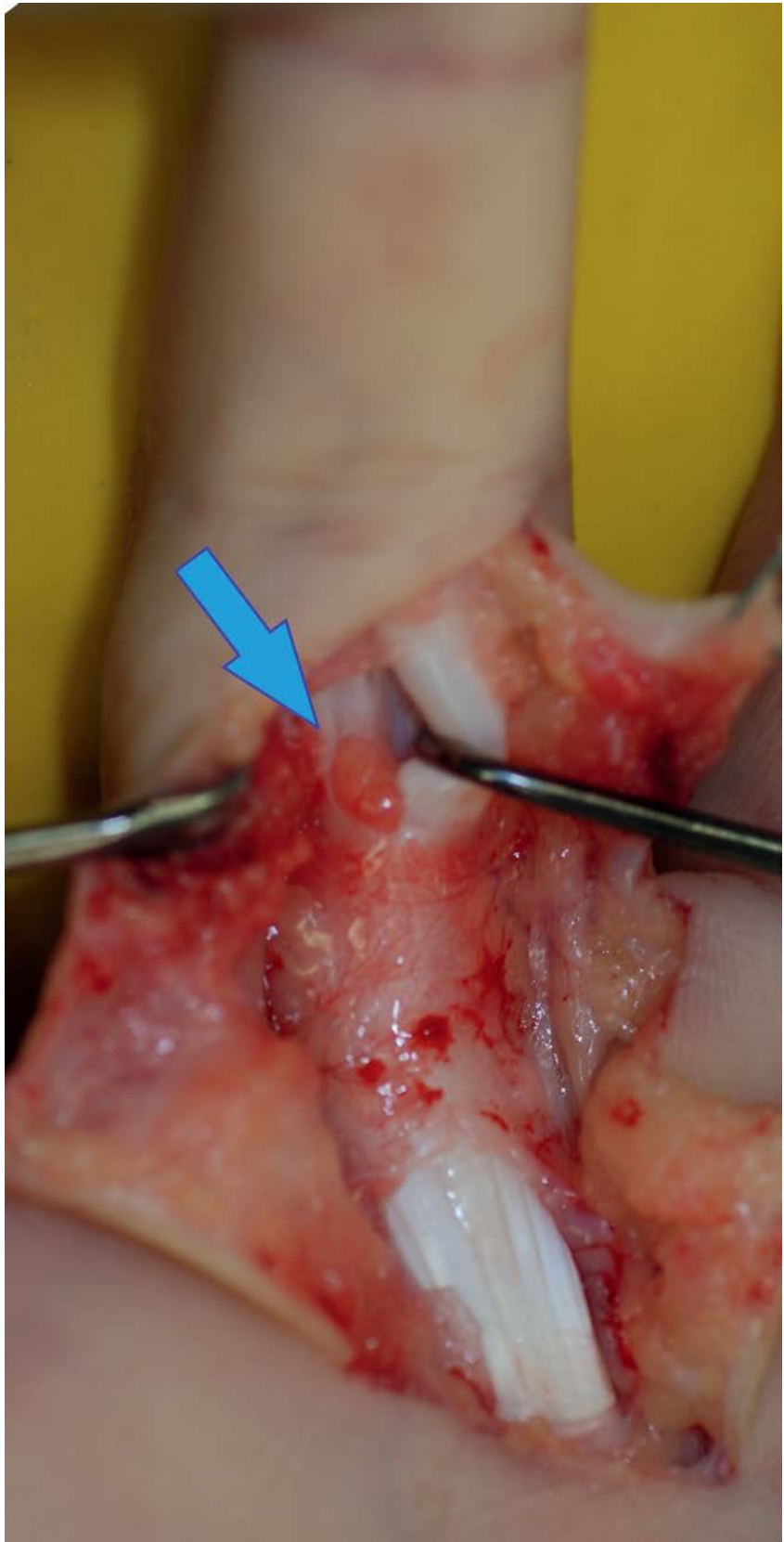

Abb. 2 Lokalisierte Synovialitis (Pfeil) zwischen FDS-Zügel und FDP-Sehne.

Missverhältnis zwischen Weite und Inhalt des osteofibrösen Gleitkanals sowohl durch eine Erweiterungsplastik des A2-Ringbandes als auch durch eine FDS-Zügelresektion beseitigt werden. Allerdings beinhaltet die A2-Erweiterungsplastik besondere Anforderungen an die Nachbehandlung. Die Anfertigung eines funktionierenden Ringbandschutzes gestaltet sich insbesondere bei kleinen Kinderfingern mit kräftigem Weichteilmantel als nahezu unmöglich. Außerdem lässt sich eine differenzierte und langdauernde Nachbehandlung bei Kleinkindern nur schwer realisieren. Von daher griffen wir die von Cardon et al. [1] angegebene Technik der Resektion eines FDS-Zügels rasch auf, die in der Nachbehandlung unkompliziert ist. Sie führt sowohl in unserem Krankengut als auch in der Literatur zu konstant guten Ergebnissen $[1,3,4]$.

Der Vergleich unserer Untersuchungsergebnisse mit der Literatur belegt die Annahme, dass die Pathophysiologie des schnellenden Fingers im Kindesalter variabler und komplexer als im 


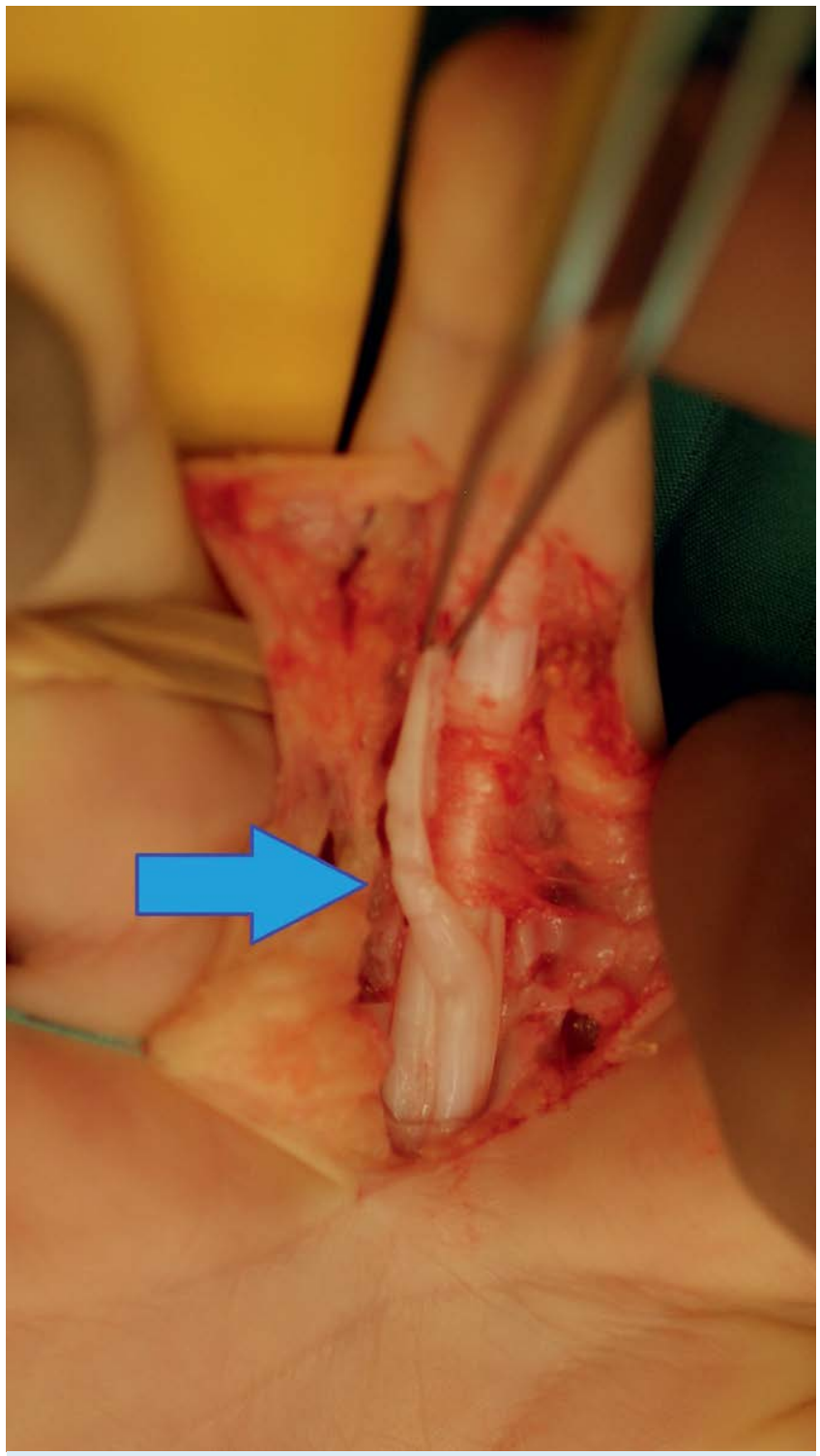

Abb. 3 Perlschnurartige Verdickungen im ulnaren FDS-Zügel.

Erwachsenenalter ist. So finden sich in der Literatur zahlreiche unterschiedliche Pathologien, die für die Symptomatik eines schnellenden Fingers verantwortlich gemacht werden. Neben den oben angegebenen intraoperativen Befunden werden auch abnorme Verbindungen zwischen der oberflächlichen und der tiefen Beugesehne sowie eine abnorme Ausformung des Chiasma tendinum beschrieben $[1,4,5]$. Diese ätiologischen Faktoren, die sich mit unseren Erfahrungen decken, können natürlich auch in Kombination auftreten. $\odot$ Abb. 5 zeigt beispielhaft eine knotige Verdickung in der tiefen Beugesehne proximal des A2-Ringbandes mit fortbestehender Gleitbehinderung nach A1-Ringbandspaltung. Gehäuft treten schnellende Finger im Kindesalter auch bei der Mucopolysaccharidose [6] und bei entzündlichen Erkrankungen wie der juvenilen rheumatoiden Arthritis oder der kalzifizierenden Tendinitis [7] auf. Diese Vielfalt der möglichen Pathologien erfordert präoperativ eine exakte Analyse und intraoperativ eine Beseitigung aller zur Pathologie beitragenden Faktoren.

Muss jede festgestellte Tendovaginosis stenosans der Finger im Kindesalter sofort operativ behandelt werden? Die Aussagen in der Literatur in Bezug auf den Spontanverlauf und den Ergebnis-

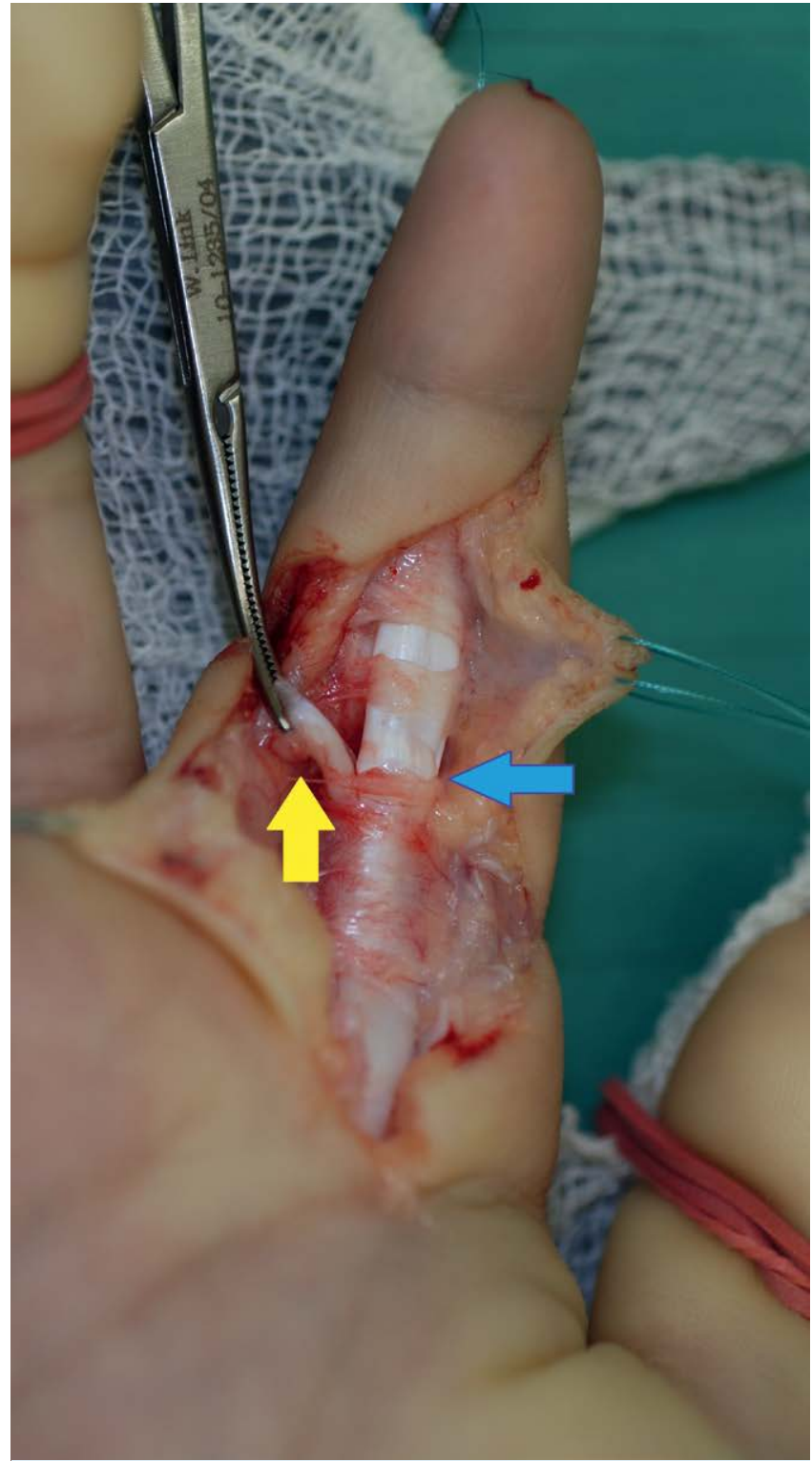

Abb. 4 Knotige Verdickung in der FDP-Sehne (blauer Pfeil); Resektion des ulnaren FDS-Zügels (gelber Pfeil)

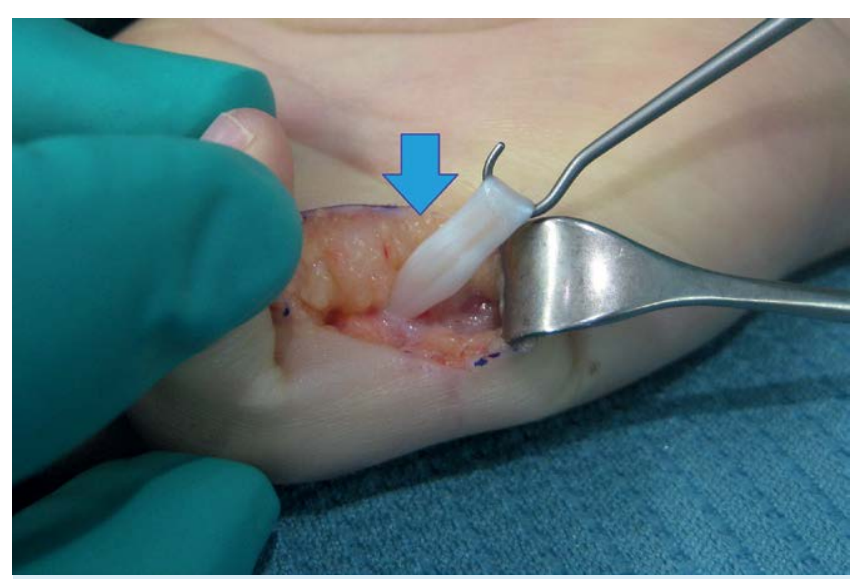

Abb. 5 Knotige Verdickung der FDP-Sehne proximal des A2-Ringbandes mit persistierender Gleitbehinderung nach A1-Ringbandspaltung. 
sen nach konservativer Therapie sind widersprüchlich. Bei den vorliegenden Publikationen handelt es sich um retrospektive Analysen, in denen oft der schnellende Finger und der Pollex rigidus gemeinsam betrachtet werden. Das Krankheitsbild des schnellenden Fingers im Kindesalter ist einfach zu selten, um valide Daten generieren zu können. Shah und Bae stellen beim Blick auf die Literatur angesichts berichteter Spontanheilungsraten zwischen 0 und $100 \%$ bei geringen Fallzahlen in retrospektiven Studien den Informationsgehalt solcher Arbeiten völlig in Frage [8]. Aus den vorliegenden Arbeiten kann man ableiten, dass grundsätzlich zwar eine Spontanheilung möglich ist. Allerdings bleibt nicht vorhersehbar, bei welchen Patienten diese tatsächlich eintritt. Darüber hinaus ist der klinische Verlauf langwierig und Rezidive sind möglich [9].

Umstritten ist auch der Stellenwert konservativer Therapieformen. Der positive Effekt einer Schienenbehandlung ist in der Literatur beschrieben $[10,11]$. In einer kürzlich publizierten Studie bestätigen Shiozawa et al. [9] den positiven Effekt einer Schienenbehandlung im Vergleich zum Spontanverlauf. Man muss allerdings bedenken, dass die Autoren die durchschnittliche Behandlungsdauer bis zur Symptombeseitigung mit 10 Monaten (Minimum: 5 Monate, Maximum: 5 Jahre) angeben. In der Gruppe ohne Schienentherapie trat eine beträchtliche Anzahl von Rezidiven auf. Es mussten letztlich $29 \%$ der Patienten mit Schienentherapie und 65\% der Patienten ohne Schienentherapie doch operiert werden [9]. Mit Waters und Bae [3] favorisieren wir die operative Behandlung des schnellenden Fingers. Es spricht nichts gegen einen konservativen Behandlungsversuch, sei es einfach durch Beobachten des Spontanverlaufs oder durch Schienenbehandlung. In der Literatur wird die Indikation zur operativen Therapie mehrheitlich nach 6 Monaten erfolgloser konservativer Therapie gesehen [2,3,12]. Manche Autoren operieren grundsätzlich [1]; andere setzen kein zeitliches Limit mit dem Argument, dass durch eine konservative Schienentherapie keine irreversiblen zusätzlichen Schäden entstehen und jederzeit die operative Therapie durchgeführt werden kann [9]. Wir meinen, dass die Indikationsstellung zur Operation individuell mit den Eltern nach Besprechen der Datenlage erfolgen sollte. Sie hängt stark von der Einstellung der Eltern gegenüber operativen Maßnahmen, der Bereitschaft zu langwierigen und im Ergebnis unsicheren konservativen Behandlungsmaßnahmen sowie von der funktionellen Beeinträchtigung durch den schnellenden Finger $a b$.

Entschließt man sich zur operativen Behandlung, so haben wir gezeigt, dass die einfache Spaltung des Ringbandes A1 im Gegensatz zur Situation beim Erwachsenen nicht immer ausreichend ist. Bei der Planung des operativen Eingriffes kann bereits die sorgfältige klinische Untersuchung Hinweise auf die Genese des Schnappphänomens geben. So weist ein tastbarer Knoten im Beugesehnenverlauf proximal des A1-Ringbandes auf die klassische Situation einer A1-Ringbandstenose hin, während ein tastbarer Knoten weiter peripher zwischen A1- und A2-Ringband oder am distalen Rand des A2-Ringbandes eher auf eine Problematik in Höhe des A2-Ringbandes hindeutet. Im Vorfeld der geplanten Operation ist es wichtig, den Eltern die potentielle Komplexität der Veränderung zu erklären und nicht den Eindruck einer Bagatellerkrankung zu erwecken.

OP-Technik: Der operative Eingriff selbst beginnt mit einer schrägen Inzision über der Region des A1-Ringbandes, die dann im Bedarfsfall zick-zack-förmig nach peripher verlängert wer-

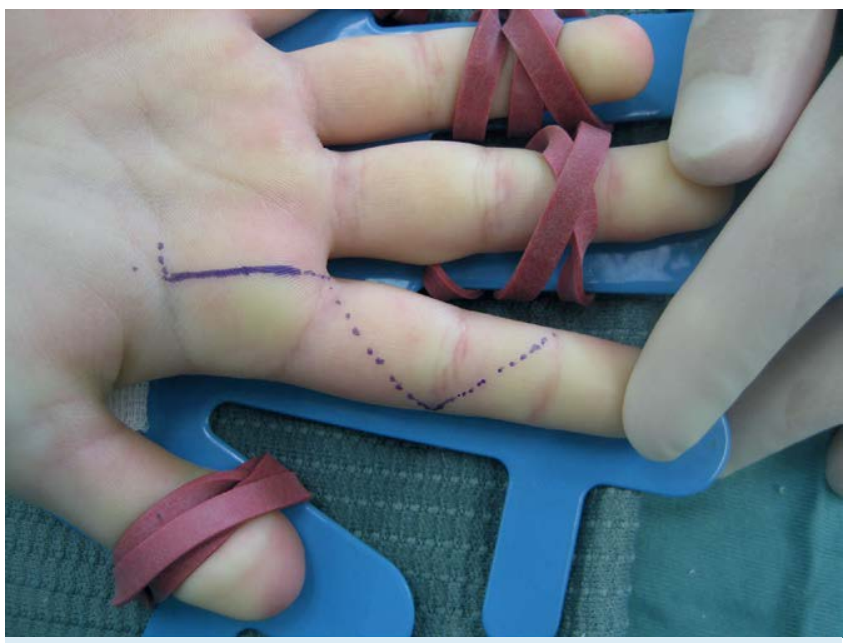

Abb. 6 Inzision zur Spaltung des Ringbandes A1 (durchgezogene Linie) mit. zick-zack-förmiger Erweiterungsmöglichkeit im Bedarfsfall (gepunktete Linie)

den kann ( $\bullet$ Abb. 6). Nach Exposition des A1-Ringbandes erfolgt die Beurteilung pathologischer Veränderungen. Nach vollständiger Spaltung des Ringbandes A1 wird überprüft, ob die Beugesehnen frei gleiten. Dies geschieht durch passives Durchbewegen des Fingers und durch Zug an der oberflächlichen und tiefen Beugesehne mittels eines Sehnenhakens. Die Beugesehnen werden auf pathologische Veränderungen überprüft. Besteht der Verdacht auf eine verbliebene Behinderung des Gleitvorgangs muss eine Schnitterweiterung nach peripher erfolgen. Da das Schnappen durch ein Missverhältnis zwischen dem Inhalt und der Weite des osteofibrösen Gleitkanals unter dem A2- oder sogar A3-Ringband ausgelöst wird, muss mehr Raum geschaffen werden, wobei eine Insuffizienz des A2-Ringbandes unbedingt vermieden werden muss. Dies ist bei einer Synovialitis durch die Synovialektomie möglich. Kann die Gleitbehinderung nicht behoben werden, bevorzugen wir wie Cardon et al. [1] die Resektion eines Zügels der oberflächlichen Beugesehne. Dabei resezieren wir bei pathologischen Veränderungen in einem FDS-Zügel natürlich den pathologisch veränderten, anderenfalls einen etwaig hypoplastischer ausgebildeten, ansonsten den ulnaren Zügel. Die Spaltung des A3-Ringbandes erscheint im Bedarfsfall unproblematisch, wird von van Heest sogar generell zusätzlich zur Spaltung des Ringbandes A1 und der Resektion des ulnaren FDS-Zügels empfohlen [12]. Auch eine begrenzte Einkerbung am A2-Ringband erscheint in besonderen Situationen vertretbar, sofern die Funktion des A2-Ringbandes im Wesentlichen erhalten bleibt. Bei der Therapie versuchen wir die von Schaverien et al. [5] erwähnte Erweiterungsplastik des A2-Ringbandes so irgend möglich zu vermeiden, um der Gefahr eines klinisch relevanten Bogensehnenphänomens vorzubeugen. Genau auf diesem Phänomen beruht auch der einzige krasse Misserfolg bei unseren Patienten. Bei der Patientin, die eine Beugekontraktur im Mittelgelenk von $90^{\circ}(\diamond$ Abb. 1) entwickelte, war neben der Spaltung des Ringbandes A1 und der Resektion eines FDS-Zügels eine komplette Spaltung des Ringbandes A2 mit anschließender Rekonstruktion mit dem FDS-Anteil erfolgt.

Am Ende des Eingriffes muss sich der Operateur nochmals von der freien Gleitfähigkeit beim passiven Durchbewegen und der Prüfung mit dem Sehnenhaken überzeugen. Unser Therapiekonzept ist grafisch im Flussdiagramm 1 zusammengefasst. 


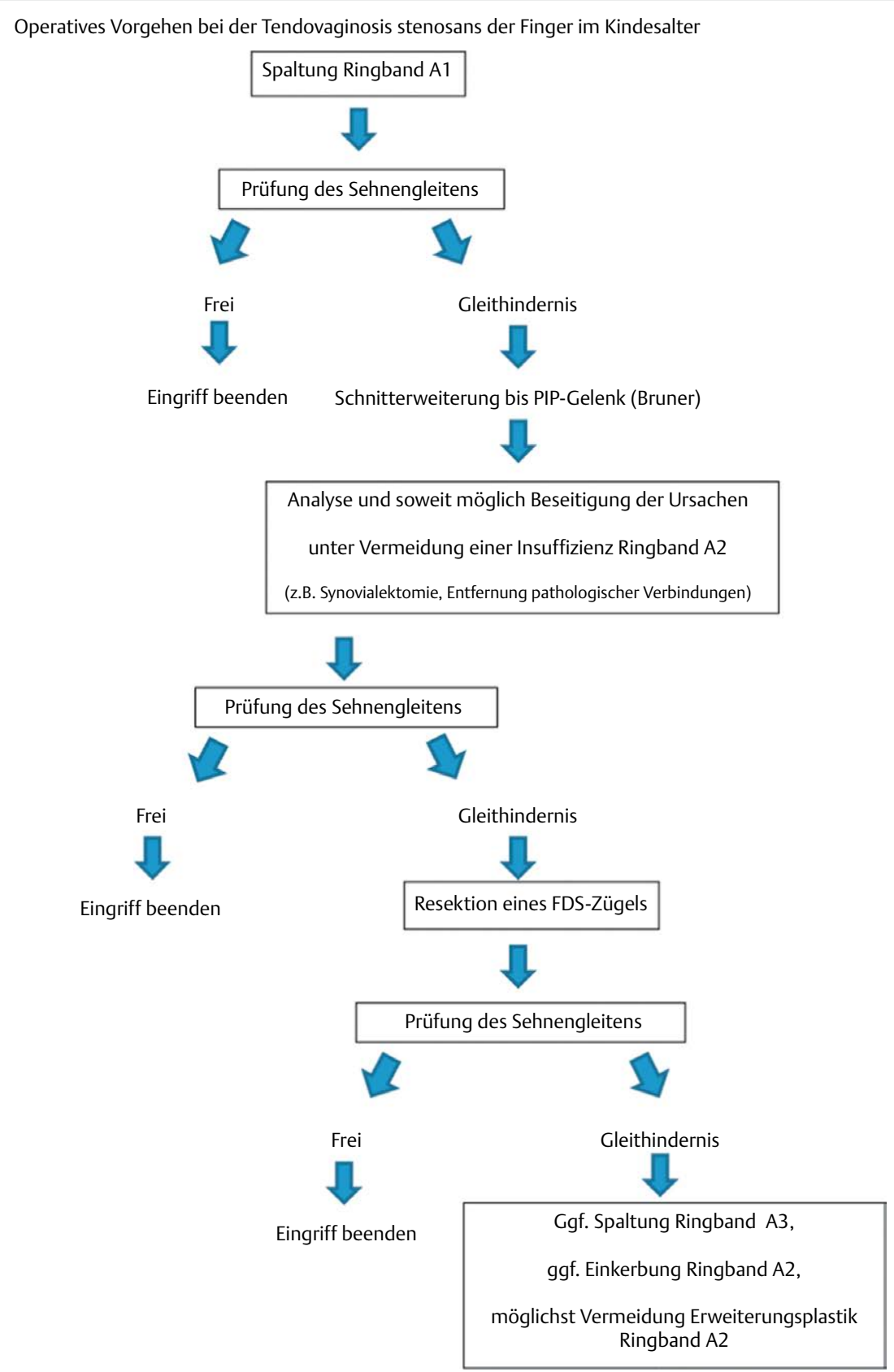

Flussdiagramm 1 Operatives Vorgehen bei der Tendovaginosis stenosans der Finger im Kindesalter
In der Nachbehandlung sollte man sich von der freien Funktion und normalem Einsatz des Fingers überzeugen. Bei Tendenz zur Schonung ist auch bei kleinen Kindern eine kurzzeitige Anleitung durch einen Handtherapeuten hilfreich.

Die vorliegende Arbeit hat Limitierungen. Die Datenerhebung erfolgte retrospektiv mit Erfassung von Patienten in 2 handchirurgischen Zentren über einen Zeitraum von mehr als 10 Jahren. Die Nachuntersuchung konnte nur in Form einer telefonischen Befragung durchgeführt werden. Allerdings war es nur so möglich, bei diesem seltenen Krankheitsbild Daten einer vernünftigen Zahl an Patienten auswerten zu können.

\section{Schlussfolgerung}

Der schnellende Finger im Kindesalter ist selten und weist sowohl im Vergleich zum schnellenden Finger des Erwachsenen als auch zum wesentlich häufigeren Pollex rigidus therapierelevante Besonderheiten in Form einer variablen Pathophysiologie auf. Therapeutisch sind konservative Maßnahmen langwierig und im Ergebnis unsicher. Die Ergebnisse nach operativer Therapie sind gut. Allerdings ist die alleinige Spaltung des Ringbandes A1 in einem beträchtlichen Prozentsatz unzureichend. Intraoperativ müssen die Sehnen auf pathologische Veränderungen überprüft werden. Ein fortbestehendes Schnappphänomen nach A1-Ringbandspaltung liegt im Missverhältnis zwischen Inhalt und Weite des osteofibrösen Gleitkanals begründet. Kann das Schnappen nicht durch Spaltung des Ringbandes A1 behoben werden, ist die Resektion eines FDS-Sehnenzügels effektiv, um mehr Raum im osteofibrösen Gleitkanal zu schaffen. Mit diesem Behandlungskonzept sollte die Funktionsstörung dauerhaft $\mathrm{zu}$ beseitigen sein. 


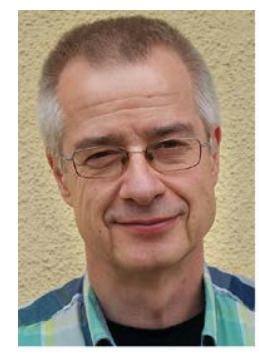

Dr. med. Karlheinz Kalb

Geb. am 5. 8. 1958 in Schweinfurt. Medizinstudium an der Universität Würzburg von 1977 bis 1984. Promotion 1986 an der Universität Würzburg. Nach Ableistung des Wehrdienstes erste chirurgische Weiterbildungsstelle 1985-1986 am SANA Krankenhaus in Wildbad/Schwarzwald (Dr. W. Strauss, Dr. H. Kalchschmidt). Von 1986 bis 1992 Ausbildung an der Chirurgischen Universitätsklinik Würzburg (Prof. Dr. E. Kern, Prof. Dr. A. Thiede). 1992 Anerkennung als Chirurg. Ab 1992 Assistenzarzt an der Klinik für Handchirurgie, Bad Neustadt/Saale (Prof. Dr. B. Landsleitner). 1997 Zusatzbezeichnung „Handchirurgie“. Seit 2000 ordentliches Mitglied der DGH. 2002 „European Diploma in Hand Surgery“. Seit 2002 Oberarzt der Klinik für Handchirurgie, Bad Neustadt/Saale (Prof. Dr. U. Lanz, Prof. Dr. K.-J. Prommersberger, Prof. Dr. J. van Schoonhoven). Seit 2012 Mitglied des European Board of Hand Surgery Diploma Examination der FESSH. Seit 2014 Mitglied des wissenschaftlichen Beirats der Zeitschrift „Handchirurgie, Mikrochirurgie, Plastische Chirurgie“.

Interessenkonflikt: Nein.

\section{Literatur}

1 Cardon LJ, Ezaki M, Carter PR. Trigger finger in children. J Hand Surg Am 1999; 24: 1156-1161

2 Tordai P, Engkvist O. Trigger fingers in children. J Hand Surg Am 1999; 24: $1162-1165$

3 Waters PM, Bae DS. Trigger Digits. In: Waters PM, Bae DS.editors. Pediatric Hand and Upper Limb surgery - A practical guide. Philadelphia: Lippincottt Williams \& Wilkins, a Wolters Kluwer business; 2012: p $114-120$

4 Bae DS, Sodha S, Waters PM. Surgical treatment of the pediatric trigger finger. J Hand Surg Am 2007; 32: 1043-1047

5 Schaverien MV, Godwin Y. Paediatric trigger finger: Literature review and management algorithm. J Plast Reconstr Aesthet Surg 2011; 64: 623-631

6 Van Heest AE, House J, Krivit W et al. Surgical treatment of carpal tunnel syndrome and trigger digits in children with mucopolysaccharide storage disorders. J Hand Surg Am 1998; 23: 236-243

7 Seiler JG 3rd, Kerwin GA. Adolescent trigger finger secondary to post-traumatic chronic calcific tendinitis. J Hand Surg Am 1995; 20: $425-427$

8 Shah AS, Bae DS. Management of pediatric trigger thumb and trigger finger. J Am Acad Orthop Surg 2012; 20: 206-213

9 Shiozawa R, Uchiyama S, Sugimoto $Y$ et al. Comparison of splinting versus nonsplinting in the treatment of pediatric trigger finger. J Hand Surg Am 2012; 37: 1211-1216

10 Nemoto $K$, Nemoto $T$, Terada $N$ et al. Splint therapy for trigger thumb and finger in children. J Hand Surg Br 1996; 21: 416-418

11 Tsuyuguchi $Y$, Tada K, Kawaii $H$. Splint therapy for trigger finger in children. Arch Phys Med Rehabil 1983; 64: 75-76

12 Van Heest AE. Pediatric Trigger Thumb and Finger. In: Abzug JM, Kozin S, Zlotolow DA. (Eds.). The Pediatric Upper Extremity. 3. New York: Springer Science and Business Media; 2015: p 1735-1742 